Draft version OCtober 31, 2018

Preprint typeset using $\mathrm{LAT}_{\mathrm{E}} \mathrm{X}$ style emulateapj v. 08/22/09

\title{
DISCOVERY OF STRONG RADIATIVE RECOMBINATION CONTINUA FROM THE SUPERNOVA REMNANT IC 443 WITH SUZAKU
}

\author{
H. Yamaguchi ${ }^{1}$, M. Ozawa ${ }^{2}$, K. Koyama ${ }^{2}$, K. Masai $^{3}$, J. S. Hiraga ${ }^{1}$, M. Ozaki ${ }^{4}$, And D. Yonetokd \\ Draft version October 31, 2018
}

\begin{abstract}
We present the Suzaku spectroscopic study of the Galactic middle-aged supernova remnant (SNR) IC 443. The X-ray spectrum in the $1.75-6.0 \mathrm{keV}$ band is described by an optically-thin thermal plasma with the electron temperature of $\sim 0.6 \mathrm{keV}$ and several additional Lyman lines. We robustly detect, for the first time, strong radiative recombination continua (RRC) of $\mathrm{H}$-like $\mathrm{Si}$ and $\mathrm{S}$ around at 2.7 and $3.5 \mathrm{keV}$. The ionization temperatures of Si and $\mathrm{S}$ determined from the intensity ratios of the RRC to He-like $\mathrm{K} \alpha$ line are $\sim 1.0 \mathrm{keV}$ and $\sim 1.2 \mathrm{keV}$, respectively. We thus find firm evidence for an extremely-overionized (recombining) plasma. As the origin of the overionization, a thermal conduction scenario argued in previous work is not favored in our new results. We propose that the highly-ionized gas were made at the initial phase of the SNR evolution in dense regions around a massive progenitor, and the low electron temperature is due to a rapid cooling by an adiabatic expansion.

Subject headings: ISM: individual (IC 443) — supernova remnants — radiation mechanisms: thermal — X-rays: ISM
\end{abstract}

\section{INTRODUCTION}

IC 443 (G189.1+3.0), a Galactic supernova remnant (SNR) at a distance of $1.5 \mathrm{kpc}$ (Welsh \& Sallmen 2003), is located near the Gem OB1 association and a dense giant molecular cloud (Cornett et al. 1977) with OH maser emission (Claussen et al. 1997). These facts strongly suggest that the remnant originated from a collapse of a massive progenitor. Braun \& Strom (1986) proposed that the shock wave has expanded into the pre-existing wind-blown bubble shell. This was confirmed by a kinematical study of the optical filaments (Meaburn et al. 1990). A comprehensive X-ray study of IC 443 was first made with the Einstein and HEAO-A2 satellites (Petre et al. 1988). They estimated the SNR age to be $\sim 3000 \mathrm{yr}$. Recently, Troja et al. (2008) derived the age of $\sim 4000 \mathrm{yr}$ from the morphologies of the shocked ejecta and interstellar medium (ISM) revealed by XMM-Newton. Thus, IC 443 is a middle-aged SNR.

Using ASCA, Kawasaki et al. (2002) found that the ionization degrees of $\mathrm{Si}$ and $\mathrm{S}$ were significantly higher than those expected from the electron temperature of the bremsstrahlung continuum. Therefore, it was argued that the plasma is in overionization $\left(k T_{z}>k T_{e}\right.$, where $k T_{z}$ and $k T_{e}$ are ionization and electron temperatures, respectively). On the other hand, Troja et al. (2008) found that the plasma is in collisional ionization equilibrium (CIE), or the overionization is only marginal based on XMM-Newton data. These two controversial results hinge upon small differences, if any, between the esti-

\footnotetext{
Electronic address: hiroya@crab.riken.jp

${ }^{1}$ RIKEN (The Institute of Physical and Chemical Research), 2 1 Hirosawa, Wako, Saitama 351-0198, Japan

2 Department of Physics, Kyoto University, Kitashirakawaoiwake-cho, Sakyo-ku, Kyoto 606-8502, Japan

3 Department of Physics, Tokyo Metropolitan University, 1-1 Minami-Osawa, Hachioji, Tokyo 192-0397, Japan

${ }^{4}$ Institute of Space and Astronautical Science, JAXA, 3-1-1 Yoshinodai, Sagamihara, Kanagawa 229-8510, Japan

${ }^{5}$ Department of Physics, Kanazawa University, Kakuma-machi, Kanazawa, Ishikawa 920-1192, Japan
}

mated electron and ionization temperatures.

In this letter, we investigate whether the overionized plasma is really present or not, by utilizing the superior spectral capabilities for diffuse sources of X-ray Imaging Spectrometers (XIS: Koyama et al. 2007) aboard the Suzaku satellite (Mitsuda et al. 2007). If present, we will study the plasma condition quantitatively to discuss the possible origin of the overionization.

\section{OBSERVATION AND DATA REDUCTION}

The northern part of IC 443 was observed with Suzaku on 2007 March 6 (Observation ID = 501006010). Three XISs located at the foci of the independent X-Ray Telescopes (XRT: Serlemitsos et al. 2007) were operating ${ }^{6}$. Two of the XISs are Front-Illuminated (FI) CCDs and the other is a Back-Illuminated (BI) CCD. The XIS was operated in the normal full-frame clocking mode with a spaced-row charge injection technique (Uchiyama et al. 2009) during the observation. For the data reduction and spectral analysis, we used the HEADAS software package of version 6.5 and XSPEC version 11.3.2, respectively. The XIS data of revision 2.0 were employed, but reprocessed using the xispi software and the latest calibration database released on 2009 February 3. After the screening with the standard criteria $^{7}$, an effective exposure of $\sim 42 \mathrm{ksec}$ was obtained.

\section{ANALYSIS AND RESULTS}

Figure 1 shows the vignetting-corrected XIS image in $1.75-3.0 \mathrm{keV}$, the energy band including the major lines of K-shell emissions from $\mathrm{Si}$ and $\mathrm{S}$. We extracted the spectrum from the brightest region, the rectangular with an angular size of $7^{\prime} \times 5^{\prime}$ (see Fig. 1). This approximately corresponds to the "Center" region of Kawasaki et al. (2002). The XIS spectra of the two FIs in the entire energy range were made by subtracting the non X-ray

\footnotetext{
${ }^{6}$ Suzaku carried four XISs, but one is now out of operation due to a damage possibly by an impact of a micro-meteorite

7 http://heasarc.nasa.gov/docs/suzaku/processing/criteria_xis.html
} 


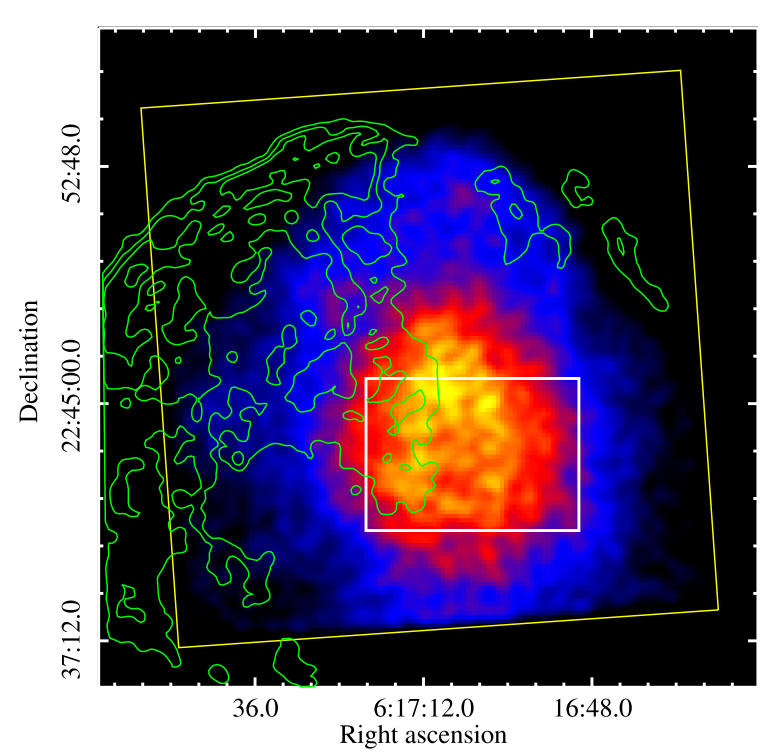

FIG. 1.- Vignetting-corrected XIS image of the northern part of IC 443 in the $1.75-3.0 \mathrm{keV}$ band, shown on an intensity color scale. The coordinates (R.A. and Dec.) refer to epoch J2000.0. The data from the three active XISs are combined and smoothed with a Gaussian kernel of $\sigma=25^{\prime \prime}$. The yellow square and the white rectangle indicate the XIS field of view and the region used in our spectral analysis, respectively. The optical Digitized Sky Survey image is overplotted in contour.

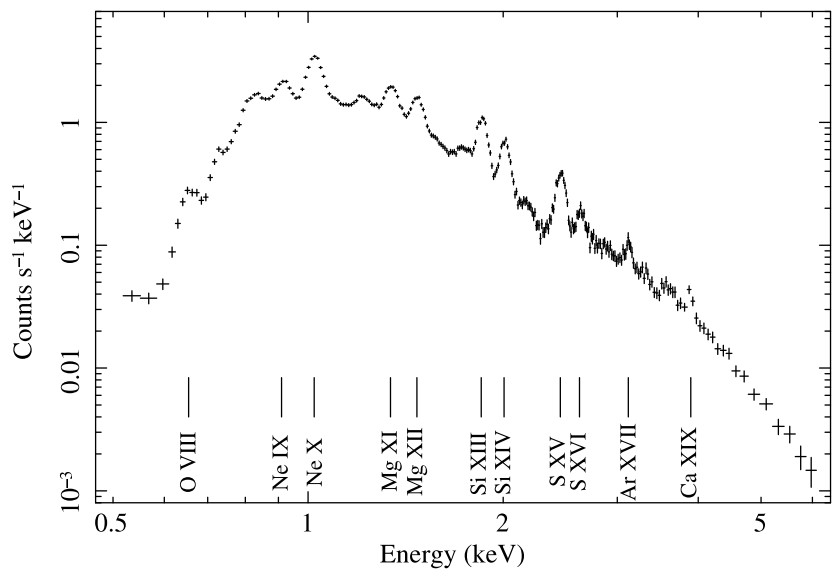

FIG. 2. - Full-band XIS-FI spectrum where the NXB is subtracted. The energies of prominent $\mathrm{K} \alpha$ emission lines from specific elements are labeled in the panel.

background (NXB) constructed with the xisnxbgen software. The spectra were merged to improve the statistics, because the response functions are almost identical each other. Figure 2 shows the resultant spectrum. We can see several prominent lines of $\mathrm{K} \alpha$ emission from He- and H-like ions (hereafter, $\operatorname{He} \alpha$ and $\operatorname{Ly} \alpha$ ). The centroids of the Ly $\alpha$ lines were measured with a Gaussian line model, and compared with the canonical values of the Astrophysical Plasma Emission Database (APED: Smith et al. 2001). The averaged center energy difference was $+4 \mathrm{eV}$. Therefore, we added a $4 \mathrm{eV}$ offset in the FI spectrum. The BI spectrum was made with the same procedure as the FIs, but an offset of $-10 \mathrm{eV}$ was added to correct the energy scale. In order to examine the ionization states of $\mathrm{Si}$ and $\mathrm{S}$, we hereafter focus on the spectrum in the energy range above $1.75 \mathrm{keV}$ (Fig. 3). Detailed studies
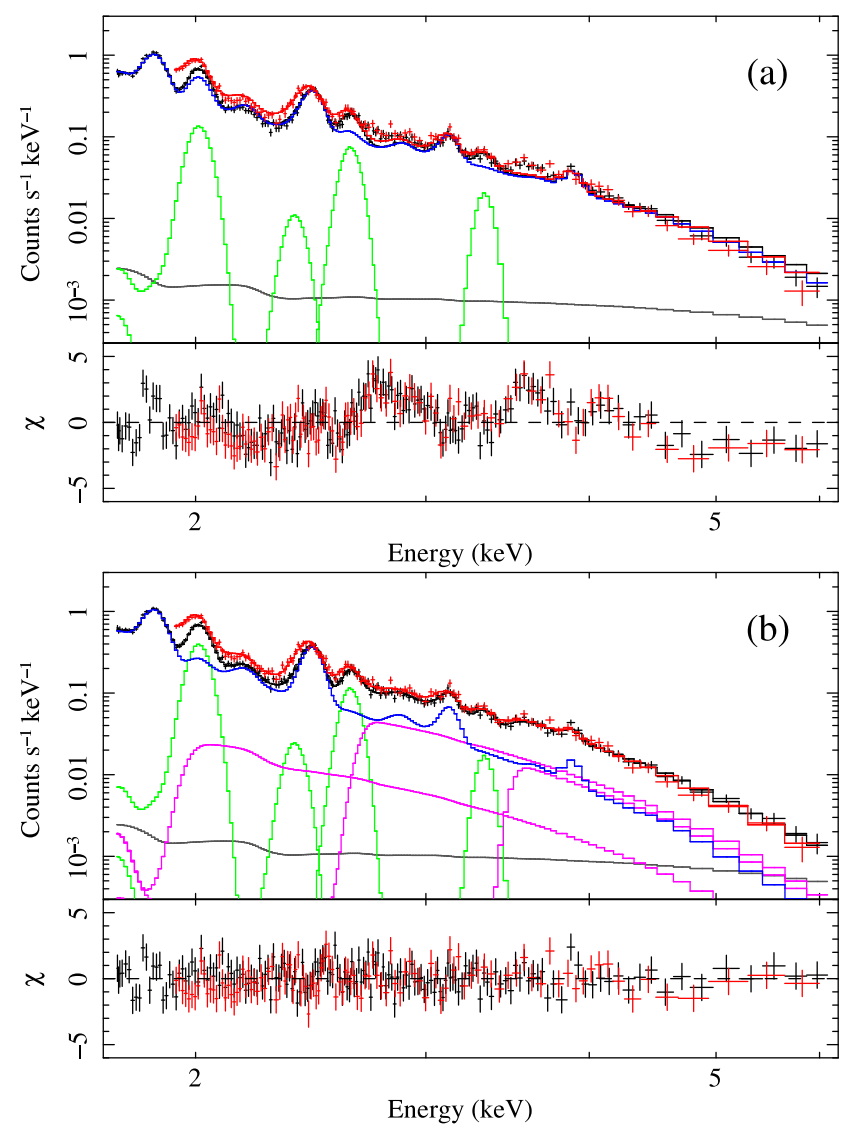

FIG. 3.- (a) XIS spectrum in the 1.75-6.0 keV band. Black and red represent FI and BI, respectively. Individual components of the best-fit model for the FI data are shown with solid colored lines: blue, green, and gray are the VAPEC, Gaussians (Si-Ly $\alpha, \mathrm{Si}-$ Ly $\beta$, S-Ly $\alpha$, and Ar-Ly $\alpha$ ), and CXB, respectively. The lower panel shows the residual from the best-fit model. Two hump-like features are clearly found around the energies of $\sim 2.7 \mathrm{keV}$ and $\sim 3.5 \mathrm{keV}$. (b) Same spectrum as (a), but for a fit with RRC components of $\mathrm{H}$-like $\mathrm{Mg}, \mathrm{Si}$, and $\mathrm{S}$ (magenta lines). The residuals seen in (a) are disappeared.

including the lower energies will be reported in a separate paper.

We first fitted the spectrum with a model of a thinthermal plasma in CIE state (a VAPEC model). The abundances (Anders \& Grevesse 1989) of Si, S, and Ar were free parameters, while the $\mathrm{Ca}$ abundance was tied to Ar. Interstellar extinction was fixed to a hydrogen column density of $N_{\mathrm{H}}=7 \times 10^{21} \mathrm{~cm}^{-2}$ with the solar elemental abundances, following Kawasaki et al. (2002) and Troja et al. (2008). The cosmic X-ray background (CXB) spectrum was approximated by a power-law model with photon index of $\Gamma=1.412$ and the surface brightness in the $2-10 \mathrm{keV}$ band of $6.4 \times 10^{-8} \mathrm{erg} \mathrm{cm}^{-2} \mathrm{~s}^{-1} \mathrm{sr}^{-1}$ (Kushino et al. 2002). Since IC 443 is located in the anti-Galactic center direction, contribution of the Galactic ridge $\mathrm{X}$-ray emission was ignored. In the initial fit, we found a significant inconsistency between the FI and BI data around the energy of neutral Si K-edge (1.84 keV). This is due to the well-known calibration issue of the XIS. Since the calibration for the FI CCDs is currently far better than the BI, we decided to ignore the energy band below $1.9 \mathrm{keV}$ in the BI spectrum. This fit leaved further large residuals, in both spectra, at the energies of S and Ar Ly $\alpha$ lines, and hence was rejected with the 
TABLE 1

BEST-Fit SPECTRAL PARAMETERS

\begin{tabular}{|c|c|c|c|}
\hline Component & \multicolumn{2}{|c|}{ Parameter } & Value \\
\hline \multirow[t]{5}{*}{ CIE (VAPEC) } & \multicolumn{2}{|c|}{$k T_{e}(\mathrm{keV})$} & $0.61(0.59-0.64)$ \\
\hline & \multicolumn{2}{|l|}{$Z_{\mathrm{Si}}($ solar $)$} & $0.82(0.78-0.85)$ \\
\hline & \multicolumn{2}{|l|}{$Z_{\mathrm{S}}($ solar $)$} & $1.7(1.6-1.8)$ \\
\hline & \multicolumn{2}{|c|}{$Z_{\mathrm{Ar}}($ solar $)$} & $2.5(2.2-2.8)$ \\
\hline & \multicolumn{2}{|c|}{$\operatorname{VEM}^{\mathrm{a}}\left(10^{12} \mathrm{~cm}^{-5}\right)$} & $6.4(6.3-6.6)$ \\
\hline & \multicolumn{3}{|c|}{ Additional components } \\
\hline & \multicolumn{2}{|c|}{$E E^{E(\mathrm{keV})^{\mathrm{t}}}$} & Flux $^{\mathrm{c}}$ \\
\hline \multirow[t]{4}{*}{ Line } & Si Ly $\alpha$ & 2.006 & $2.8(2.7-2.9)$ \\
\hline & Si Ly $\beta$ & 2.377 & $0.21(0.14-0.29)$ \\
\hline & $\mathrm{S} \operatorname{Ly} \alpha$ & 2.623 & $0.84(0.79-0.90)$ \\
\hline & $\operatorname{Ar} \operatorname{Ly} \alpha$ & 3.323 & $0.11(0.085-0.14)$ \\
\hline \multirow[t]{3}{*}{$\mathrm{RRC}$} & H-like $\mathrm{Mg}$ & 1.958 & $1.2(1.0-1.5)$ \\
\hline & H-like Si & 2.666 & $2.2(2.0-2.3)$ \\
\hline & H-like S & 3.482 & $0.46(0.41-0.51)$ \\
\hline
\end{tabular}

Note. - The uncertainties in the parentheses are the $90 \%$ confidence range. ${ }^{a}$ Volume emission measure, $\int n_{e} n_{p} d V /\left(4 \pi D^{2}\right)$, where $n_{e}, n_{p}, V$, and $D$ are the electron and proton densities $\left(\mathrm{cm}^{-3}\right)$, the emitting volume $\left(\mathrm{cm}^{3}\right)$, and the distance to the source $(\mathrm{cm})$, respectively. ${ }^{b}$ The fixed energy values of the line center or the K-edge. ${ }^{c}$ Total flux in the unit of $10^{-4}$ photon $\mathrm{cm}^{-2} \mathrm{~s}^{-1}$.

$\chi^{2} /$ dof of $935 / 270$.

We, therefore, applied a model of one-temperature VAPEC (CIE plasma) plus narrow Gaussian lines at 2006, 2623, and $3323 \mathrm{eV}$, the Ly $\alpha$ energies of the $\mathrm{Si}$, $\mathrm{S}$, and Ar, hydrogenic ions, respectively. This process is essentially the same as Kawasaki et al. (2002). Ly $\beta$ lines of the same elements were also added, but they were not significant except for $\mathrm{Si}(2377 \mathrm{eV})$. The result is shown in Figure 3a, with the best-fit CIE plasma temperature of $k T_{e} \sim 0.94 \mathrm{keV}$. Although the $\chi^{2} /$ dof value was significantly reduced to $657 / 266$, the model was still unacceptable. In fact, apparent hump-like residuals are found around $\sim 2.7 \mathrm{keV}$ and $\sim 3.5 \mathrm{keV}$, in addition to the systematic model excess in the continuum at the energies above $\sim 4.5 \mathrm{keV}$.

We checked systematic error due to the CXB fluctuation by allowing the CXB intensity as a free parameter. The fit, however, did not improve at all. We also tried two-component VAPEC models with independent temperatures and abundances, but these were rejected with $\chi^{2} /$ dof $=737 / 265$. The results were essentially the same as figure $3 \mathrm{a}$; the hump-like residuals remained at $\sim 2.7 \mathrm{keV}$ and $\sim 3.5 \mathrm{keV}$. No additional CIE component nor non-equilibrium ionization (NEI) plasma model removed the hump-like residuals with significant improvement of the $\chi^{2}$ value.

At the energies of the humps, no emission line candidate from an abundant element is found. However, the energies are consistent with the K-shell binding potentials $\left(I_{z}\right)$ of the H-like $\mathrm{Si}(2666 \mathrm{eV})$ and $\mathrm{S}(3482 \mathrm{eV})$. Therefore, the humps are likely due to the free-bound transitions to the K-shell of the H-like Si and S. A formula for the spectrum of radiative recombination continuum (RRC) is found in equation (21) of Smith \& Brickhouse $(2002)^{8}$. When the electron temperature is much lower than the K-edge energy $\left(k T_{e} \ll I_{z}\right)$, this formula is approximated as;

$$
\frac{d P}{d E}\left(E_{\gamma}\right) \propto \exp \left(-\frac{E_{\gamma}-I_{z}}{k T_{e}}\right), \text { for } E_{\gamma} \geq I_{z}
$$

8 http://cxc.harvard.edu/atomdb/physics/plasma/plasma.html

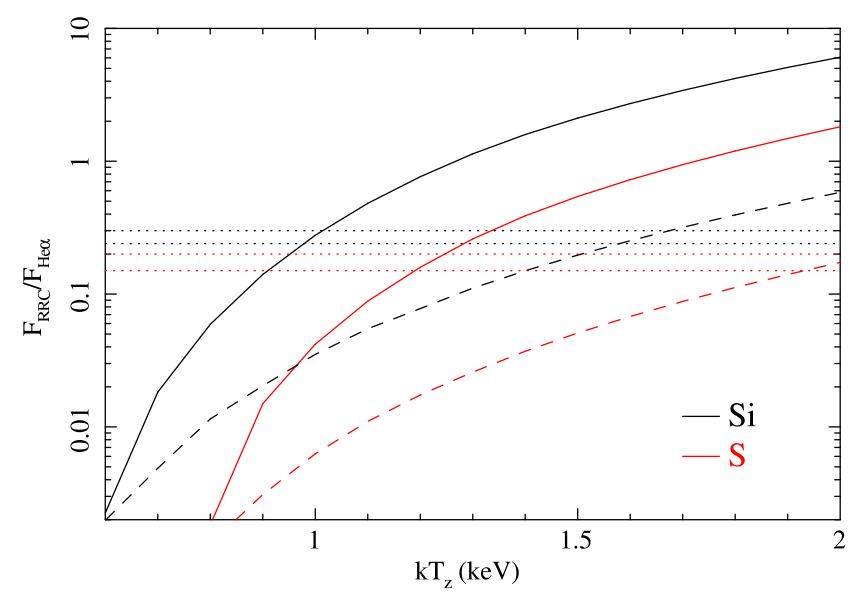

FIG. 4-- Emissivity ratios of H-like RRC to He-like $\mathrm{K} \alpha$ lines as a function of ionization temperature $\left(k T_{z}\right)$, predicted by the plasma radiation code of Masai (1994) for recombining plasma with electron temperature $\left(k T_{e}\right)$ of $0.6 \mathrm{keV}$. Black and red solid lines represent $\mathrm{Si}$ and $\mathrm{S}$, respectively. For comparison, the same ratios for CIE $\left(k T_{z}=k T_{e}\right)$ plasma (the APEC model: Smith 2001) are indicated by dashed lines. The horizontal dotted lines represent the $90 \%$ upper and lower limits of the observed values.

Thus, the width of the RRC structure depends on the electron temperature. We added the RRC of Equation 1 for $\mathrm{H}_{-}$like $^{9} \mathrm{Si}$ and $\mathrm{S}$. The $k T_{e}$ values of the RRC were linked to that of the VAPEC component. Then, the fit was dramatically improved to an acceptable $\chi^{2} /$ dof of $290 / 264$. Although the hump-like residuals were completely removed by this fit, we further added the RRC model of $\mathrm{H}$-like $\mathrm{Mg}$. This step is reasonable because $\mathrm{Mg}$ is more abundant than $\mathrm{Si}$ and $\mathrm{S}$ in the solar abundance ratio of Anders \& Grevesse (1989) and the K-edge energy of H-like $\mathrm{Mg}\left(I_{z}=1958 \mathrm{eV}\right)$ falls into the analyzed band. The $\chi^{2} /$ dof value was significantly reduced to $267 / 263$, which gives an $F$-test probability of $\sim 3 \times 10^{-6}$. The best-fit parameters and model are given in Table 1 and Figure 3b, respectively. The spectrum was also fitted with the independent electron temperatures for the VAPEC and RRC components, but the values are consistent with each other within their statistical uncertainties.

\section{DISCUSSION AND CONCLUSION}

We have found that the $1.75-6.0 \mathrm{keV}$ spectrum cannot be represented with CIE nor NEI plasma alone, but need the additional fluxes of the Lyman lines and the H-like RRC of Si and S (and possibly $\mathrm{Mg}$ ). This is the first detection of clear RRC emissions in an SNR. In the following, we quantitatively discuss the implications of our spectral results.

From the best-fit model in Table 1, the flux ratios of H-like RRC to He-like $\mathrm{K} \alpha(\mathrm{He} \alpha)$ line $\left(F_{\mathrm{RRC}} / F_{\mathrm{He} \alpha}\right)$ are given to be $0.28(0.24-0.30)$ and $0.18(0.15-0.20)$ for $\mathrm{Si}$ and $\mathrm{S}$, respectively. These are compared, in Figure 4. with the modeled emissivity ratios by the plasma radiation code of Masai (1994) for the electron temperature of $0.6 \mathrm{keV}$. We find that the large observed ratios of $F_{\mathrm{RRC}} / F_{\mathrm{He} \alpha}$ are significantly above those in the CIE case $\left(k T_{z}=k T_{e}\right)$, but can be reproduced in the overionization case $\left(k T_{z}>k T_{e}\right)$. The ionization temperatures of $\mathrm{Si}$ and $\mathrm{S}$ are determined to be $\sim 1.0 \mathrm{keV}$ and $\sim 1.2 \mathrm{keV}$,

9 "H-like RRC" refers to the free-bound emission due to electron captures by fully-ionized ions into ground state of $\mathrm{H}$-like ions 
respectively. This is, therefore, the firm evidence of the overionized (recombining) plasma.

The overionization claim for IC 443 was first argued by Kawasaki et al. (2002). Using $A S C A$ data, they derived $k T_{z}$ to be $\sim 1.5 \mathrm{keV}$ from the $\mathrm{S} \operatorname{Ly} \alpha / \mathrm{He} \alpha$ flux ratio compared with the predicted emissivity ratio in the CIE plasma code. Troja et al. (2008) adopted the same analysis procedure to the XMM-Newton spectrum, and claimed that $k T_{z}$ obtained from the line flux ratio was nearly same as the bremsstrahlung temperature $\left(k T_{e}\right)$. However, since the RRC process is accompanied with electron captures to the excited levels, as given in Equation 3 below, the resulting cascade decay to the ground state contributes the line emission. In a CIE plasma, on the other hand, origins of the line emissions are more dominated by the collisional excitation. Therefore, a $k T_{z}$ determination done by comparing with a CIE plasma code is not a proper method. Also, the previous works assumed that the continuum spectrum purely consists of bremsstrahlung emission, and determined $k T_{e}$ to be $\sim 1.0 \mathrm{keV}$. This value has been reduced owing to the discovery of the strong RRC emissions.

It should be noted that the elemental abundances in Table 1 are determined as the parameters of the $0.6 \mathrm{keV}$ VAPEC (CIE) component, and hence should be modified in the real case of the overionized plasma. The intensity of the Si-He $\alpha$ line is given as $F_{\mathrm{He} \alpha} \propto \varepsilon\left(T_{e}\right) \cdot f_{\mathrm{He}}\left(T_{z}\right) \cdot Z_{\mathrm{Si}}$, where $\varepsilon\left(T_{e}\right)$ and $f_{\mathrm{He}}\left(T_{z}\right)$ are, respectively, emissivity coefficient for electron temperature $T_{e}$ and fraction of He-like ion for ionization temperature $T_{z}$. The abundance, therefore, can be modified by the fraction ratio of He-like ions, $f_{\mathrm{He}}(0.6 \mathrm{keV}) / f_{\mathrm{He}}(1.0 \mathrm{keV})$. According to the ionization population calculations by Mazzotta et al. (1998), the ion fractions of He-like, H-like, and fully-ionized $\mathrm{Si}\left(f_{\mathrm{He}}, f_{\mathrm{H}}, f_{0}\right)$ are estimated to be $(0.83$, $0.15,0.01)$ for $k T_{z}=0.6 \mathrm{keV}$ and $(0.37,0.43,0.19)$ for $k T_{z}=1.0 \mathrm{keV}$, respectively. Then, the modified $Z_{\mathrm{Si}}$ is $0.82 \times(0.83 / 0.37) \simeq 2.2$ solar. For $\mathrm{S},\left(f_{\mathrm{He}}, f_{\mathrm{H}}, f_{0}\right)$ are $(0.91,0.02,0)$ for $k T_{z}=0.6 \mathrm{keV}$ and $(0.59,0.32,0.06)$ for $k T_{z}=1.2 \mathrm{keV}$. Thus, we similarly modified $Z_{\mathrm{S}}$ to be $1.7 \times(0.91 / 0.59) \simeq 2.6$ solar .

Using the measured RRC flux, we can independently determine the volume emission measure, $\mathrm{VEM}=$ $\int n_{e} n_{p} d V /\left(4 \pi D^{2}\right)$, from a following equation:

$$
F_{\mathrm{RRC}}=\alpha_{1}\left(T_{e}\right) \cdot n_{Z} / n_{p} \cdot f_{0} \cdot \mathrm{VEM},
$$

where $\alpha_{1}\left(T_{e}\right)$ and $n_{Z}$ are $\mathrm{K}$-shell $\mathrm{RRC}$ rate coefficient for electron temperature $T_{e}$ and number density of element $Z$, respectively. According to Badnell (2006), we find total radiative recombination rate for fully-ionized $\mathrm{Si}$, at $k T_{e}=0.6 \mathrm{keV}$, is $\alpha_{\text {tot }} \sim 2.3 \times 10^{-12} \mathrm{~cm}^{3} \mathrm{~s}^{-1}$. Since the rate of the recombination into a level of principal quantum number $n$ is described as;

$$
\alpha_{n} \propto \frac{1}{n^{3}}\left(\frac{3}{2} \frac{k T_{e}}{I_{z}}+\frac{1}{n^{2}}\right)^{-1}
$$

(e.g., Nakayama \& Masai 2001), we obtain $\alpha_{1} / \alpha_{\text {tot }}=$ 0.65 , for $k T_{e}=0.6 \mathrm{keV}$ and $I_{z}=2.666 \mathrm{keV}$. Thus, the $\mathrm{VEM}$ is calculated to be $\sim 9.9 \times 10^{12}\left(Z_{\mathrm{Si}} / 2.2 \text { solar }\right)^{-1}$ $\left(f_{0} / 0.19\right)^{-1} \mathrm{~cm}^{-5}$. Similarly, $\alpha_{\text {tot }}$ at $0.6 \mathrm{keV}$ for fullyionized $\mathrm{S}$ is derived to be $\sim 3.2 \times 10^{-12} \mathrm{~cm}^{3} \mathrm{~s}^{-1}$. Therefore, the RRC flux of $\mathrm{S}$ corresponds to the VEM of $\sim 9.4 \times 10^{12}\left(Z_{\mathrm{S}} / 2.6 \text { solar }\right)^{-1}\left(f_{0} / 0.06\right)^{-1} \mathrm{~cm}^{-5}$. These values are almost consistent with that of the VAPEC component (Table 1).

The rectangular region in Figure 1 corresponds to $3.1 \times 2.2 \mathrm{pc}^{2}$ at $D=1.5 \mathrm{kpc}$. Assuming the plasma depth of $3 \mathrm{pc}$, the emitting volume is estimated to be $6.1 \times 10^{56} \mathrm{~cm}^{3}$. Therefore, the VEM of $6.6 \times 10^{12} \mathrm{~cm}^{-5}$ is converted to the uniform electron density of $n_{e} \sim$ $1.7 \mathrm{~cm}^{-3}$.

As the mechanism to form the overionized plasma, Kawasaki et al. (2002) proposed that the SNR consists of a central hot $\left(k T_{e} \sim 1.0 \mathrm{keV}\right)$ region surrounded by a cool $\left(k T_{e} \sim 0.2 \mathrm{keV}\right)$ outer shell, and interpreted that the hot interior cooled down via thermal conduction to the cool exterior. Under the several reasonable boundary conditions, they estimated the cooling time from $k T_{e}$ $=1.5 \mathrm{keV}$ to $1.0 \mathrm{keV}$ is about $(3-10) \times 10^{3} \mathrm{yr}$, roughly the same as the SNR age ( $\sim 4000$ yr: Troja et al. 2008). However, this scenario cannot work on our new results. According to equation (5) of Kawasaki et al. (2002), the conduction timescale is estimated to be

$$
t_{\text {cond }} \simeq 3.9 \times 10^{4}\left(\frac{n_{e}}{1.7 \mathrm{~cm}^{-3}}\right)\left(\frac{k T_{e}}{0.6 \mathrm{keV}}\right)^{-5 / 2} \mathrm{yr},
$$

if the similar boundary condition is assumed. Thus, cooling via conduction requires far longer time than the SNR age. Photo-ionization is also unlikely because no strong ionizing source is found. Furthermore, the temperature of $\sim 0.6 \mathrm{keV}$ is significantly higher than that of a typical photo-ionization plasma $(\lesssim 0.1 \mathrm{keV}$ : e.g., Kawashima \& Kitamoto 1996).

Since the progenitor of IC 443 has been suggested to be a massive star with strong stellar wind activity (Braun \& Strom 1986; Meaburn et al. 1990), we propose another possibility that the rapid and drastic cooling is due to a rarefaction process, as discussed by Itoh \& Masai (1989). If a supernova explodes in a dense circumstellar medium made in the progenitor's super giant phase, the gas is shock-heated to high temperature and significantly ionized at the initial phase of the SNR evolution. Subsequent outbreak of the blast wave to a low-density ISM caused drastic adiabatic expansion of the shocked gas and resultant rapid cooling of the electrons. The lifetimes of the fully-stripped ions are roughly estimated to be $\tau=\left(\alpha_{\mathrm{tot}} n_{e}\right)^{-1} \simeq 8.1 \times 10^{3}\left(n_{e} / 1.7 \mathrm{~cm}^{-3}\right)^{-1}$ yr for $\mathrm{Si}$ and $\simeq 5.8 \times 10^{3}\left(n_{e} / 1.7 \mathrm{~cm}^{-3}\right)^{-1}$ yr for $\mathrm{S}$, respectively. Note that these values are underestimated compared to the actual timescale for the plasma to reach CIE, because the contribution of collisional ionization processes is ignored. Nevertheless, the estimated lifetimes are longer than the age of IC 443. The overionized plasma can, therefore, still survive at present.

Future observations with very high energy resolution like the Astro- $H$ mission will give firm evidence for the RRC structure not only on Si and S but also on the other major elements. This will provide more quantitative study on the peculiar SNR IC 443.

The authors deeply appreciate the referee, Randall Smith, for his constructive suggestions on revising manuscript. We also acknowledge helpful discussions with Kazuo Makishima and Aya Bamba. H. Yamaguchi and J. S. Hiraga are supported by the Special Postdoctoral Researchers Program in RIKEN. M. Ozawa is 
a Research Fellow of Japan Society for the Promotion of Science (JSPS). This work is partially supported by the Grant-in-Aid for the Global COE Program "The Next Generation of Physics, Spun from Universality and
Emergence", Young Scientists (HY), and Challenging Exploratory Research (KK) from the Ministry of Education, Culture, Sports, Science and Technology (MEXT) of Japan.

\section{REFERENCES}

Anders, E., \& Grevesse, N. 1989, Geochim. Cosmochim. Acta, 53 , 197

Badnell, N. R. 2006, ApJS, 167, 334

Braun, R., \& Strom, R. G. 1986, A\&A, 164, 193

Claussen, M. J., Frail, D. A., Goss, W. M., \& Gaume, R. A. 1997 ApJ, 489, 143

Cornett, R. H., Chin, G., \& Knapp, G. R. 1977, A\&A, 54, 889

Itoh, H., \& Masai, K. 1989, MNRAS, 236, 885

Kawashima, K., \& Kitamoto, S. 1996, PASJ, 48, L113

Kawasaki, M. T., Ozaki, M., Nagase, F., Masai, K., Ishida, M., \& Petre, R. 2002, ApJ, 572, 897

Koyama, K., et al. 2007, PASJ, 59, 23

Kushino, A., Ishisaki, Y., Morita, U., Yamasaki, N. Y., Ishida, M., Ohashi, T., \& Ueda, Y. 2002, PASJ, 54, 327

Masai, K. 1994, ApJ, 437, 770

Mazzotta, P., Mazzitelli, G., Colafrancesco, S., \& Vittorio, N. 1998, A\&AS, 133, 403
Meaburn, J., Whitehead, M. J., Raymond, J. C., Clayton, C. A., \& Marston, A. P. 1990, A\&A, 227, 191

Mitsuda, K., et al. 2007, PASJ, 59, 1

Nakayama, M., \& Masai, K. 2001, A\&A, 375, 328

Petre, R., Szymkowiak, A. E., Seward, F. D., \& Willingale, R. 1988, ApJ, 335, 215

Serlemitsos, P. J., et al. 2007, PASJ, 59, 9

Smith, R. K., Brickhouse, N. S., Liedahl, D. A., \& Raymond, J. C. 2001, ApJ, 556, L91

Troja, E., Bocchino, F., Miceli, M., \& Reale, F. 2008, A\&A, 485, 777

Uchiyama, H., et al. 2009, PASJ, 61, 9

Welsh, B. Y., \& Sallmen, S. 2003, A\&A, 408, 545 